\title{
REPRESENTATION OF QUANTUM STATES AS POINTS IN A PROBABILITY SIMPLEX ASSOCIATED TO A SIC-POVM
}

\author{
JOSÉ IGNACIO ROSADO
}

\begin{abstract}
The quantum state of a $d$-dimensional system can be represented by a probability distribution over the $d^{2}$ outcomes of a Symmetric Informationally Complete Positive Operator Valued Measure (SIC-POVM), and then this probability distribution can be represented by a vector of $\mathbb{R}^{d^{2}-1}$ in a $\left(d^{2}-1\right)$-dimensional simplex, we will call this set of vectors $\mathcal{Q}$. Other way of represent a $d$-dimensional system is by the corresponding Bloch vector also in $\mathbb{R}^{d^{2}-1}$, we will call this set of vectors $\mathcal{B}$. In this paper it is proved that with the adequate scaling $\mathcal{B}=\mathcal{Q}$. Also we indicate some features of the shape of $\mathcal{Q}$.
\end{abstract}

\section{INTRODUCTION}

In quantum mechanics a quantum state is described by a density operator, $\rho$, but there are alternative descriptions. One alternative description is to parameterize the space of density matrices with Bloch vectors and study the structure formed by these vectors, $\mathcal{B}$, this is done thoroughly in [6] and 7 . Other possible description is provided by the probabilities $p_{i}=\operatorname{Tr}\left(E_{i} \rho\right)$, where $E_{i}$ are the elements of a SICPOVM [8, an informationally complete and symmetric POVM, with the minimal number of elements. This is the description of quantum states chosen in QuantumBayesianism [3].

According to the quantum-Bayesian approach to quantum foundations, see for example 4, probabilities $p_{i}$ represent an agents Bayesian degrees of belief. When we represent the probabilities of a SIC-POVM as points in the corresponding probability simplex, $\Delta$, we will see that these probabilities are not arbitrary, not any point of $\Delta$ can represent a quantum state, only a proper subset $\mathcal{Q} \subset \Delta$. The problem is then to understand the structure of $\mathcal{Q}$, if it is possible, in physical terms. Why are the beliefs of our agent constrained in this way? I think a hint to the answer to this question is to realize that the structure of $\mathcal{Q}$ is agent independent, of course for a particular experiment two agents can differ in the assignments of probabilities for the outcomes of a SIC-POVM, but the two distributions will be represented by points in $\mathcal{Q}$. Then the structure of $\mathcal{Q}$ is saying us something about the external world, at least about the intersubjective world.

The main result of this paper is demonstrate that $\mathcal{B}=\mathcal{Q}$, with suitable scalings. We therefore can translate the results obtained in the study of $\mathcal{B}$ to probability concepts, and I think this is a useful translation because in terms of probabilities we can made use of the tools of Quantum Information Theory and our physical intuition in trying to understand the why of the $\mathcal{Q}$ structure.

The structure of this paper is as follows. In SECTIOn 2 we review some basic facts about simplexes as geometrical objects that can represent probability distributions. In SECTION 3 we review the Bloch representation of quantum states and the conditions these vectors satisfy. In SECTION 4 we give the Bloch representation

Date: November 1, 2018.

Key words and phrases. Bloch vectors, probability simplex, SIC-POVM's. 
of a SIC-POVM, and see what conditions satisfy the corresponding Bloch vectors. In Section 5 we construct $\mathcal{Q}$ and demonstrate its equality with $\mathcal{B}$, we also give some features of the shape of this set.

\section{Simplexes AND PROBABILITy Distributions}

This section contains known facts about simplexes and probability distributions, but serves to collect useful results and to fix some conventions in this paper.

Definition 1. An n-dimensional and regular simplex in $\mathbb{R}^{n}$ is any set, $\Delta_{n+1}$, that can be defined as follows

$$
\begin{aligned}
\Delta_{n+1}= & \left\{\mathbf{s}=\sum_{i=1}^{n+1} p_{i} \mathbf{t}_{i}: p_{i} \in[0,1], \sum_{i=1}^{n+1} p_{i}=1, \mathbf{t}_{i} \in \mathbb{R}^{n}\right. \text { and } \\
& \left.\mathbf{t}_{i} \cdot \mathbf{t}_{j}=(a-b) \delta_{i j}+b, \quad i, j \in\{1,2, \ldots, n+1\} \text { and } b \neq a \neq 0\right\} .
\end{aligned}
$$

That is, $\Delta_{n+1}$ is the convex hull of the set of vectors $V=\left\{\mathbf{t}_{i}\right\}_{i=1}^{n+1}$, the position vectors of the vertices of $\Delta_{n+1}$.

Some observations about this definition:

First: Definition 1 provides a bijective map from probability distributions over $n+1$ outcomes, $\left\{p_{i}\right\}_{i=1}^{n+1}$, to $\Delta_{n+1}$.

Second: $\mathbf{t}_{i}^{2}=a$, all vertices are at equal distance from the origin, a necessary condition to obtain a regular simplex centered at the origin, and this distance is not zero so that the simplex is not a point.

Third: $\mathbf{t}_{i} \cdot \mathbf{t}_{j}=b$ when $i \neq j$, this implies that the vectors of $V$ spread uniformly in space. The condition $b \neq a$ is necessary so that the vectors in $V$ are different. Also we can deduce that $b \neq 0$ because we cannot have $n+1$ orthogonal and no null vectors in $\mathbb{R}^{n}$.

Now we will deduce some important properties.

Proposition 1. Every subset of $V$ that contains $n$ vectors is a basis of $\mathbb{R}^{n}$.

Proof. Given the symmetry between the vectors of $V$ we can, without loss of generality, prove the proposition for the subset $\left\{\mathbf{t}_{i}\right\}_{i=1}^{n}$. Therefore we have to prove that

$$
\sum_{i=1}^{n} \lambda_{i} \mathbf{t}_{i}=\mathbf{0}
$$

has only the trivial solution. We multiply (1) by $\mathbf{t}_{n+1}$ and the result is

$$
b \sum_{i=1}^{n} \lambda_{i}=0
$$

and because $b \neq 0$

$$
\sum_{i=1}^{n} \lambda_{i}=0
$$


Now we multiply (1) by $\mathbf{t}_{j}$, where $j \in\{1,2, \ldots, n\}$

$$
\begin{aligned}
\sum_{i=1}^{n} \lambda_{i} \mathbf{t}_{i} \cdot \mathbf{t}_{j} & =0 & & \\
\sum_{i=1}^{n} \lambda_{i}\left[(a-b) \delta_{i j}+b\right] & =0 & & \\
(a-b) \lambda_{j}+b \sum_{i=1}^{n} \lambda_{i} & =0 & & \\
(a-b) \lambda_{j} & =0 & & \text { (by equation (2) }) \\
\lambda_{j} & =0, & & \text { (because } a \neq b)
\end{aligned}
$$

Proposition 2. The sum of all the elements of $V$ is null.

Proof. By Proposition 1 we know that $S=\left\{\mathbf{t}_{i}\right\}_{i=1}^{n}$ is a basis of $\mathbb{R}^{n}$, so we can express $\mathbf{t}_{n+1}$ as a linear combination of S-vectors

$$
\mathbf{t}_{n+1}=\sum_{i=1}^{n} \lambda_{i} \mathbf{t}_{i}
$$

Multiplying (3) by $\mathbf{t}_{n+1}$ we obtain

$$
a=b \sum_{i=1}^{n} \lambda_{i}
$$

Now we multiply (3) by $\mathbf{t}_{j}$, with $j \in\{1,2, \ldots, n\}$

$$
\begin{aligned}
& b=\sum_{i=1}^{n} \lambda_{i}\left[(a-b) \delta_{i j}+b\right] \\
& b=(a-b) \lambda_{j}+a \quad \text { (by equation (44)), }
\end{aligned}
$$

and now is immediate that

$$
\lambda_{j}=-1, \quad j \in\{1,2, \ldots, n\} .
$$

Substituting in (3)

or

$$
\mathbf{t}_{n+1}=-\sum_{i=1}^{n} \mathbf{t}_{i}
$$

$$
\sum_{i=1}^{n+1} \mathbf{t}_{i}=\mathbf{0} .
$$

Corollary 1. The relation between $a$ and $b$ is

$$
b=-\frac{a}{n} \text {. }
$$

Proof. If in equation (4) we substitute the values found for $\lambda_{i}$ we immediately obtain (5).

Then $a$ remains as a free parameter that fixes the scale of $\Delta_{n+1}$. In view of the last corollary I think that a convenient value for $a$ is $a=n \Rightarrow b=-1$ because then many $1 / n$ factors dissapear. With these values the inner product between vectors of $V$ reads as follows

$$
\mathbf{t}_{i} \cdot \mathbf{t}_{j}=(n+1) \delta_{i j}-1 \quad i, j \in\{1,2, \ldots, n+1\},
$$


that is, $\left\|\mathbf{t}_{i}\right\|=\sqrt{n}$ and $\mathbf{t}_{i} \cdot \mathbf{t}_{j}=-1$ when $i \neq j$.

In a regular simplex all the $\mathrm{m}$-facets, m-dimensional facets, are at the same distance from the origin.

Proposition 3. The distance from the origin to the $m$-facets is

$$
d_{m}=\sqrt{\frac{n-m}{m+1}}
$$

Proof. Because all $\mathrm{m}$-facets are equidistant from the origin we can take one particular m-facet, for example that defined by the vectors $\left\{\mathbf{t}_{i}\right\}_{i=1}^{m+1}$. The centroid of this $m$ facet is in the position

$$
\mathbf{F}_{m}=\frac{1}{m+1} \sum_{i=1}^{m+1} \mathbf{t}_{i}
$$

then

$$
\begin{aligned}
d_{m}^{2} & =\mathbf{F}_{m}^{2}=\frac{1}{(m+1)^{2}} \sum_{i, j=1}^{m+1} \mathbf{t}_{i} \cdot \mathbf{t}_{j} \\
& \left.=\frac{1}{(m+1)^{2}} \sum_{i, j=1}^{m+1}\left[(n+1) \delta_{i j}-1\right] \quad \text { (by equation (6) }\right) \\
& =\frac{n-m}{m+1}
\end{aligned}
$$

Among these distances are of special interest the radius of the inner sphere and the radius of the outer sphere. The outer sphere is the $(n-1)$-sphere centered at the origin that contains $\Delta_{n+1}$ and such that its radius is minimal, evidently its radius is the distance from the origin to the 0 -facets (the vertices of the simplex)

$$
R_{\text {out }}=d_{0}=\left\|\mathbf{t}_{i}\right\|=\sqrt{n} .
$$

The inner sphere is the $(n-1)$-sphere contained in $\Delta_{n+1}$ centered at the origin and such that its radius is maximal. From (7) we see that

$$
0=d_{n}<d_{n-1}<\cdots<d_{0}=\sqrt{n},
$$

then the inner sphere has radius

$$
R_{i n}=d_{n-1}=\frac{1}{\sqrt{n}}
$$

with a greater radius the sphere would have points situated beyond the $(n-1)$ facets, and therefore outside $\Delta_{n+1}$.

As we noted Definition 1 provides a bijective map from probability distributions over $n+1$ outcomes, $\left\{p_{i}\right\}_{i=1}^{n+1}$, to $\Delta_{n+1}$, the map is

$$
\begin{aligned}
f_{\Delta_{n+1}}: \mathcal{P}_{n+1} & \rightarrow \Delta_{n+1} \\
\left\{p_{i}\right\}_{i=1}^{n+1} & \mapsto \mathbf{s}=\sum_{i=1}^{n+1} p_{i} \mathbf{t}_{i},
\end{aligned}
$$

where we denote this map by $f_{\Delta_{n+1}}$ because the vectors $\mathbf{s}$ depends on the election of simplex $\Delta_{n+1}$, we have also introduced the symbol $\mathcal{P}_{n+1}$ to denote the set of all probability distributions over $n+1$ outcomes.

In the next proposition we will see how to recover a probability distribution from a given vector $\mathbf{s} \in \Delta_{n+1}$. 
Proposition 4. The inverse of the map defined above is

$$
\begin{aligned}
f_{\Delta_{n+1}}^{-1}: \Delta_{n+1} & \rightarrow \mathcal{P}_{n+1} \\
\mathbf{s} & \mapsto\left\{p_{i}=\frac{1}{n+1}\left(\mathbf{s} \cdot \mathbf{t}_{i}+1\right)\right\}_{i=1}^{n+1}
\end{aligned}
$$

Proof.

$$
\begin{aligned}
\mathbf{s} & =\sum_{j=1}^{n+1} p_{j} \mathbf{t}_{j} \\
\mathbf{s} \cdot \mathbf{t}_{i} & =\sum_{j=1}^{n+1} p_{j} \mathbf{t}_{j} \cdot \mathbf{t}_{i} \\
\mathbf{s} \cdot \mathbf{t}_{i} & \left.=\sum_{j=1}^{n+1} p_{j}\left[(n+1) \delta_{i j}-1\right] \quad \text { (by (마) }\right),
\end{aligned}
$$

Then

$$
p_{i}=\frac{1}{n+1}\left(\mathbf{s} \cdot \mathbf{t}_{i}+1\right)
$$

It is also interesting the following relation

Proposition 5. If $\mathbf{s} \in \Delta_{n+1}$ and $\left\{p_{i}\right\}_{i=1}^{n+1}$ is its corresponding probability distribution, then

$$
\sum_{i=1}^{n+1} p_{i}^{2}=\frac{1}{n+1}\left(\mathbf{s}^{2}+1\right)
$$

Proof.

$$
\begin{aligned}
\mathbf{s}^{2} & =\sum_{i, j=1}^{n+1} p_{i} p_{j} \mathbf{t}_{i} \cdot \mathbf{t}_{j} \\
& =\sum_{i, j=1}^{n+1} p_{i} p_{j}\left[(n+1) \delta_{i j}-1\right] \\
& =(n+1) \sum_{i=1}^{n+1} p_{i}^{2}-1
\end{aligned}
$$

From which it follows the proposition.

Corollary 2. When $\mathbf{s}^{2}=d_{m}^{2}$ then

$$
\sum_{i=1}^{n+1} p_{i}^{2}=\frac{1}{m+1}
$$

\section{Bloch REPRESENTATION OF QUANTUM STATES}

We will denote by $\mathcal{D}_{d}$ the set of density matrices of order $d$, namely

$$
\mathcal{D}_{d}=\left\{\rho \in \mathcal{M}_{d}(\mathbb{C}): \rho=\rho^{\dagger}, \rho \geq 0 \text { and } \operatorname{Tr} \rho=1\right\} .
$$

Any d-dimensional density matrix $\rho$ can be represented as 5 ]

$$
\rho=\frac{1}{d}+\sqrt{\frac{d+1}{2 d}} \mathbf{r} \cdot \boldsymbol{\sigma},
$$


where the coefficient $\sqrt{\frac{d+1}{2 d}}$ has been chosen for later convenience. If the vector, $\mathbf{r} \in \mathbb{R}^{d^{2}-1}$, in (16) is such that the corresponding $\rho$ is a true density matrix, then this vector will be called the Bloch vector associated to $\rho$. The set of all Bloch vectors in $\mathbb{R}^{d^{2}-1}$ will be denoted by $\mathcal{B}_{d^{2}-1}$. The components of the vector $\boldsymbol{\sigma}=$ $\left(\sigma_{1}, \sigma_{2}, \ldots, \sigma_{d^{2}-1}\right)$ are hermitian matrices with null trace which form a basis of the algebra $\mathfrak{s u}(d)$ and we follow the convention that scalar + square matrix is read as scalar $I+$ square matrix, where $I$ is the identity matrix of the appropriate order.

Some useful formulae involving the $\sigma_{a}$ 's are, see for example appendix 2 of [2],

$$
\begin{aligned}
{\left[\sigma_{a}, \sigma_{b}\right] } & =2 \mathrm{i} f_{a b c} \sigma_{c}, \\
\left\{\sigma_{a}, \sigma_{b}\right\} & =\frac{4}{d} \delta_{a b}+2 d_{a b c} \sigma_{c}, \\
\operatorname{Tr}\left(\sigma_{a} \sigma_{b}\right) & =2 \delta_{a b}, \\
\operatorname{Tr}\left(\sigma_{a} \sigma_{b} \sigma_{c}\right) & =2 d_{a b c}+2 \mathrm{i} f_{a b c},
\end{aligned}
$$

where we sum over repeated indices, $f_{a b c} \in \mathbb{R}$ is totally antisymmetric, $d_{a b c} \in \mathbb{R}$ is totally symmetric, traceless and is identically null when $d=2$.

In the next proposition we see what conditions have to be fulfilled by $\mathbf{r}$ so that $\rho$ is a pure state. This proposition is enunciated in [2] (eq. 8.24, p. 215) although with a different normalization for the Bloch vectors.

Proposition 6. $\rho$ is a pure state if and only if the associated Bloch vector, $\mathbf{r}$, satisfies

$$
\mathbf{r}^{2}=\frac{d-1}{d+1}
$$

and

$$
\mathbf{r} * \mathbf{r}=(d-2) \sqrt{\frac{2}{d(d+1)}} \mathbf{r},
$$

where $(\mathbf{r} * \mathbf{r})_{c}=d_{a b c} r_{a} r_{b}$

Proof. $\rho$ is a pure state if and only if $\rho^{2}=\rho$

$$
\begin{array}{rlr}
\rho^{2} & =\frac{1}{d^{2}}+\frac{d+1}{2 d} r_{a} r_{b} \sigma_{a} \sigma_{b}+\frac{1}{d} \sqrt{\frac{2(d+1)}{d}} \mathbf{r} \cdot \boldsymbol{\sigma} & \\
& =\frac{1}{d^{2}}+\frac{d+1}{4 d} r_{a} r_{b}\left\{\sigma_{a}, \sigma_{b}\right\}+\frac{1}{d} \sqrt{\frac{2(d+1)}{d}} \mathbf{r} \cdot \boldsymbol{\sigma} & \quad\left(r_{a} r_{b} \text { is symmetric in } a, b\right) \\
& =\frac{1}{d^{2}}+\frac{d+1}{4 d} r_{a} r_{b}\left[\frac{4}{d} \delta_{a b}+2 d_{a b c} \sigma_{c}\right]+\frac{1}{d} \sqrt{\frac{2(d+1)}{d}} \mathbf{r} \cdot \boldsymbol{\sigma} & \text { (by equation (18) ) }) \\
& =\frac{1}{d^{2}}+\frac{d+1}{d^{2}} \mathbf{r}^{2}+\frac{d+1}{2 d}(\mathbf{r} * \mathbf{r}) \cdot \boldsymbol{\sigma}+\frac{1}{d} \sqrt{\frac{2(d+1)}{d}} \mathbf{r} \cdot \boldsymbol{\sigma} & \\
& =\frac{1}{d}\left(\frac{1}{d}+\frac{d+1}{d} \mathbf{r}^{2}\right)+\sqrt{\frac{d+1}{2 d}}\left(\sqrt{\frac{d+1}{2 d}}(\mathbf{r} * \mathbf{r})+\frac{2}{d} \mathbf{r}\right) \cdot \boldsymbol{\sigma}
\end{array}
$$

Now we impose that this linear combination of $I_{d}$, the identity matrix of order $d$, and the $\sigma_{a}$ matrices is equal to the linear combination of these same matrices in (16). Because the $\sigma_{a}$ matrices are traceless and they satisfy (19), we see that the matrices of the set $\left\{I_{d}, \sigma_{1}, \sigma_{2}, \ldots, \sigma_{d^{2}-1}\right\}$ are orthogonal, with respect to the Hilbert-Schmidt inner product, so they are linearly independent, in fact, they form a basis of the hermitian matrices of order $d$, then two linear combinations of these matrices are equal if and only if its coefficients are equal. In our case this means 
that

$$
\begin{array}{r}
\frac{1}{d}+\frac{d+1}{d} \mathbf{r}^{2}=1 \\
\sqrt{\frac{d+1}{2 d}}(\mathbf{r} * \mathbf{r})+\frac{2}{d} \mathbf{r}=\mathbf{r}
\end{array}
$$

and

From which we obtain (21) and (22).

Equation (16) defines a bijective map

$$
\begin{aligned}
q_{\boldsymbol{\sigma}}: \mathcal{B}_{d^{2}-1} & \rightarrow \mathcal{D}_{d} \\
\mathbf{r} & \mapsto \rho=\frac{1}{d}+\sqrt{\frac{d+1}{2 d}} \mathbf{r} \cdot \boldsymbol{\sigma},
\end{aligned}
$$

We write $q_{\boldsymbol{\sigma}}$ because this map is fixed once we have chosen the basis for $\mathfrak{s u}(d)$. It is interesting to find its inverse

Proposition 7. The inverse of the map $q_{\boldsymbol{\sigma}}$ is

$$
\begin{aligned}
q_{\boldsymbol{\sigma}}^{-1}: \mathcal{D}_{d} & \rightarrow \mathcal{B}_{d^{2}-1} \\
\rho & \mapsto \mathbf{r}=\sqrt{\frac{d}{2(d+1)}} \operatorname{Tr}(\rho \boldsymbol{\sigma}) .
\end{aligned}
$$

Proof.

$$
\begin{array}{rlr}
\rho & =\frac{1}{d}+\sqrt{\frac{d+1}{2 d}} r_{a} \sigma_{a} & \\
\operatorname{Tr}\left(\rho \sigma_{b}\right) & =\operatorname{Tr}\left(\frac{1}{d} \sigma_{b}+\sqrt{\frac{d+1}{2 d}} r_{a} \sigma_{a} \sigma_{b}\right) & \\
\operatorname{Tr}\left(\rho \sigma_{b}\right) & =\sqrt{\frac{d+1}{2 d}} r_{a} \operatorname{Tr}\left(\sigma_{a} \sigma_{b}\right) & \text { (because } \sigma_{b} \text { is traceless) } \\
\operatorname{Tr}\left(\rho \sigma_{b}\right) & =\sqrt{\frac{2(d+1)}{d}} r_{b} & \text { (by (19) })
\end{array}
$$

\section{Bloch Representation of a SiC-POVM}

First we define a SIC-POVM, see for example 8

Definition 2. A set of positive operators $\left\{E_{i}\right\}_{i=1}^{d^{2}}$ is a SIC-POVM, for d-dimensional systems, if the following conditions are satisfied

$$
\begin{aligned}
E_{i} & =\frac{1}{d} \rho_{i}, \quad \text { with } \rho_{i} \text { a pure state and } i \in\left\{1,2, \ldots, d^{2}\right\} . \\
\sum_{i=1}^{d^{2}} E_{i} & =1 \\
\operatorname{Tr}\left(E_{j} E_{j}\right) & =\frac{d \delta_{i j}+1}{d^{2}(d+1)}, \quad \text { with } i, j \in\left\{1,2, \ldots, d^{2}\right\} .
\end{aligned}
$$

From this definition and from the last section we see that the elements of the SIC-POVM can be represented in the following way

$$
E_{i}=\frac{1}{d^{2}}+\frac{1}{d} \sqrt{\frac{d+1}{2 d}} \mathbf{e}_{i} \cdot \boldsymbol{\sigma} .
$$

Where each $\mathbf{e}_{i}$ satisfies (21) and (22). Our next task is to find what other conditions vectors $\mathbf{e}_{i}$ satisfy so that the corresponding operators $E_{i}$ form a SIC-POVM. 
Proposition 8. The vectors $\left\{\mathbf{e}_{i}\right\}_{i=1}^{d^{2}}$ defined in (26) are the positions of the vertices of a regular and $\left(d^{2}-1\right)$-dimensional simplex.

Proof.

$$
\begin{aligned}
\operatorname{Tr}\left(E_{i} E_{j}\right) & =\operatorname{Tr}\left[\left(\frac{1}{d^{2}}+\frac{1}{d} \sqrt{\frac{d+1}{2 d}} \mathbf{e}_{i} \cdot \boldsymbol{\sigma}\right)\left(\frac{1}{d^{2}}+\frac{1}{d} \sqrt{\frac{d+1}{2 d}} \mathbf{e}_{j} \cdot \boldsymbol{\sigma}\right)\right] \\
& =\operatorname{Tr}\left(\frac{1}{d^{4}}+\frac{d+1}{2 d^{3}} e_{i a} e_{j b} \sigma_{a} \sigma_{b}\right) \\
& =\frac{1}{d^{3}}+\frac{d+1}{d^{3}} \mathbf{e}_{i} \cdot \mathbf{e}_{j} \quad(\text { by (19) }) \\
& =\frac{d \delta_{i j}+1}{d^{2}(d+1)} \quad(\text { Imposing the last condition of (25) }) .
\end{aligned}
$$

which implies

$$
\mathbf{e}_{i} \cdot \mathbf{e}_{j}=\frac{d^{2} \delta_{i j}-1}{(d+1)^{2}},
$$

and we see that the vectors $\left\{\mathbf{e}_{i}\right\}_{i=1}^{d^{2}}$ satisfy Definition 1 with $n=d^{2}-1, a=$ $(d-1) /(d+1)$, as it should be by (21), and $b=-1 /(d+1)^{2}$.

\section{Probability distributions CORResponding to QuAntum States}

Let

$$
\rho=\frac{1}{d}+\sqrt{\frac{d+1}{2 d}} \mathbf{r} \cdot \boldsymbol{\sigma}
$$

be a quantum state, mixed or pure. We will find what is the probability distribution over the outcomes of a SIC-POVM, and what is the vector in the corresponding simplex of probability, as described in SECTIOn 2, The probability distribution is

$$
\begin{aligned}
p_{i} & =\operatorname{Tr}\left(E_{i} \rho\right) \\
& =\operatorname{Tr}\left[\left(\frac{1}{d^{2}}+\frac{1}{d} \sqrt{\frac{d+1}{2 d}} \mathbf{e}_{i} \cdot \boldsymbol{\sigma}\right)\left(\frac{1}{d}+\sqrt{\frac{d+1}{2 d}} \mathbf{r} \cdot \boldsymbol{\sigma}\right)\right] \\
& =\operatorname{Tr}\left(\frac{1}{d^{3}}+\frac{d+1}{2 d^{2}} e_{i a} r_{b} \sigma_{a} \sigma_{b}\right) \\
& =\frac{1}{d^{2}}+\frac{d+1}{d^{2}} \mathbf{e}_{i} \cdot \mathbf{r}
\end{aligned}
$$

Naming by $\mathcal{Q}_{\mathcal{P}}$ the set of all probability distributions over the outcomes of the SICPOVM $\left\{E_{i}\right\}_{i=1}^{d^{2}}$ that correspond to a quantum state, then (28) defines the following bijective map

$$
\begin{aligned}
m_{E}: \mathcal{B}_{d^{2}-1} & \rightarrow \mathcal{Q}_{\mathcal{P}} \subset \mathcal{P}_{d^{2}} \\
\mathbf{r} & \mapsto\left\{p_{i}=\frac{1}{d^{2}}+\frac{d+1}{d^{2}} \mathbf{e}_{i} \cdot \mathbf{r}\right\}_{i=1}^{d^{2}}
\end{aligned}
$$

To represent this distribution we can choose any regular simplex, $\Delta_{d^{2}}$, in $\mathbb{R}^{d^{2}-1}$, the most natural is the one defined by the vectors

$$
\mathbf{t}_{i}=(d+1) \mathbf{e}_{i} \quad i \in\left\{1,2, \ldots, d^{2}\right\},
$$

because, as we have seen, the vectors $\mathbf{e}_{i}$ define themselves a simplex, the factor is needed so that $\left\|\mathbf{t}_{i}\right\|=\sqrt{d^{2}-1}$ in accordance with the norm used in SECTION 2 for these vectors. Now we make use of the bijective map $f_{\Delta_{d^{2}}}$ defined in SECTION 2 to define the set $\mathcal{Q}=f_{\Delta_{d^{2}}}\left(\mathcal{Q}_{\mathcal{P}}\right)$, this set contains therefore all elements of $\Delta_{d^{2}}$ 
corresponding to quantum states. We then have the bijective map $\left.f_{\Delta_{d^{2}}}\right|_{\mathcal{Q}_{\mathcal{P}}}$ that we will denote by $g_{\mathcal{Q}_{\mathcal{P}}}$ and is defined therefore as

$$
\begin{aligned}
g_{\mathcal{Q}_{\mathcal{P}}}: \mathcal{Q}_{\mathcal{P}} & \rightarrow \mathcal{Q} \\
\left\{p_{i}\right\}_{i=1}^{d^{2}} & \mapsto \mathbf{s}=\sum_{i=1}^{d^{2}} p_{i} \mathbf{t}_{i} .
\end{aligned}
$$

Now we can prove the main result of this paper.

Proposition 9. The set of Bloch vectors, and the set of elements of $\Delta_{d^{2}}$ corresponding to quantum states are the same set. Namely

$$
\mathcal{B}_{d^{2}-1}=\mathcal{Q} .
$$

Proof. The map $\left(g_{\mathcal{Q}_{\mathcal{P}}} \circ m_{E}\right)$ is a bijection because it is a composition of bijections, it goes from $\mathcal{B}_{d^{2}-1} \subset \mathbb{R}^{d^{2}-1}$ to $\mathcal{Q} \subset \mathbb{R}^{d^{2}-1}$. We therefore need to prove that this map is the identity map, that is

$$
\left(g_{\mathcal{Q}_{\mathcal{P}}} \circ m_{E}\right)(\mathbf{r})=\mathbf{r} \quad \forall \mathbf{r} \in \mathcal{B}_{d^{2}-1},
$$

or, equivalently

$$
m_{E}(\mathbf{r})=g_{\mathcal{Q}_{\mathcal{P}}}^{-1}(\mathbf{r})
$$

Applying (29) and (11) we obtain

$$
\begin{gathered}
\left\{\frac{1}{d^{2}}+\frac{d+1}{d^{2}} \mathbf{e}_{i} \cdot \mathbf{r}\right\}_{i=1}^{d^{2}}=\left\{\frac{1}{d^{2}}\left(\mathbf{t}_{i} \cdot \mathbf{r}+1\right)\right\}_{i=1}^{d^{2}} \\
\left\{\frac{1}{d^{2}}\left[(d+1) \mathbf{e}_{i} \cdot \mathbf{r}+1\right]\right\}_{i=1}^{d^{2}}=\left\{\frac{1}{d^{2}}\left(\mathbf{t}_{i} \cdot \mathbf{r}+1\right)\right\}_{i=1}^{d^{2}},
\end{gathered}
$$

and this last equality is true for all $\mathbf{r} \in \mathcal{B}_{d^{2}-1}$ because $\mathbf{t}_{i}=(d+1) \mathbf{e}_{i}$.

Now we can study basic facts about the shape of $\mathcal{Q}$. We will denote by $\mathfrak{P}$ the subset of $\mathcal{Q}$ corresponding to pure states.

Corollary 3. $\mathfrak{P}$ is a subset of the $\left(d^{2}-2\right)$-sphere of radius $R_{\mathfrak{P}}=\sqrt{\frac{d-1}{d+1}}$.

Proof. Because (31) we can use (21) that gives the norm of Bloch vectors corresponding to pure states.

Corollary 4. The sphere that contains $\mathfrak{P}$ is not completely inside $\Delta_{d^{2}}$, except in the case $d=2$, then it is the inner sphere of the simplex.

Proof. We have to prove that the next inequality is true, and that is an equality only if $d=2$.

$$
\begin{aligned}
R_{\mathfrak{P}}^{2} & \geq R_{i n}^{2} & & \\
\frac{d-1}{d+1} & \geq \frac{1}{d^{2}-1} & & (\text { by }(10)) \\
d-1 & \geq \frac{1}{d-1} & & (\text { multiplying by } d+1) \\
(d-1)^{2} & \geq 1 & & (\text { multiplying by } d-1)
\end{aligned}
$$

The following result was also obtained in [1].

Proposition 10. The sphere that contains $\mathfrak{P}$ is tangent to the facets of $\Delta_{d^{2}}$ of dimension $m_{\mathfrak{P}}=\frac{(d+2)(d-1)}{2}$. 
Proof. First observe that $m_{\mathfrak{P}}$ is a natural number because $d+2$ and $d-1$ have opposite parity, so one of them is divisible by 2. Equation (17) gives de distance from the origin to the m-facets, we will demonstrate that $d_{m}$, for the particular value $m=m_{\mathfrak{P}}$, is the radius of the sphere that contains $\mathfrak{P}$.

$$
\begin{aligned}
d_{m_{\mathfrak{P}}} & =\sqrt{\frac{d^{2}-1-m_{\mathfrak{P}}}{m_{\mathfrak{P}}+1}} \\
& =\sqrt{\frac{d^{2}-1-\frac{(d+2)(d-1)}{2}}{\frac{(d+2)(d-1)}{2}+1}} \\
& =\sqrt{\frac{d-1}{d+1}}
\end{aligned}
$$

Therefore $\mathcal{Q}$ is a subset of a $\left(d^{2}-1\right)$-ball truncated by the $m$-facets of $\Delta_{d^{2}-1}$ with $m>m_{\mathfrak{P}}=\frac{(d+2)(d-1)}{2}$, because from (9) we have that if $m>m_{\mathfrak{P}}$ then $d_{m}<d_{m_{\mathfrak{P}}}$. But the shape of $\mathcal{Q}$ is not simply this truncated ball, as we have emphasized it is a proper subset of this body. Remember that the pure states are a $(2 d-2)$-dimensional manifold, so not all points on the surface of the ball, even those situated inside the simplex, can be quantum states.

The following result can be found in 3 , although it is obtained in a different way.

Corollary 5. If $\left\{p_{i}\right\}_{i=1}^{d^{2}}$ is the distribution of probability over the outcomes of a $S I C-P O V M$ of a pure state then

$$
\sum_{i=1}^{d^{2}} p_{i}^{2}=\frac{2}{d(d+1)} .
$$

Proof. We simply use Corollary 2 and the last proposition.

$$
\begin{aligned}
\sum_{i=1}^{d^{2}} p_{i}^{2} & =\frac{1}{m_{\mathfrak{P}}+1} \\
& =\frac{1}{\frac{(d+2)(d-1)}{2}+1} \\
& =\frac{2}{d(d+1)} .
\end{aligned}
$$

\section{Conclusions AND Future RESEARCH}

We have proved that with suitable scalings the set of Bloch vectors, $\mathcal{B}$, is equal to the set of points, $\mathcal{Q}$, of the simplex associated to the probability distributions over the outcomes of a SIC-POVM that correspond to quantum states. We have see that $\mathcal{Q}$ is a subset of a $d^{2}-1$-ball truncated by the $m$-facets of a $d^{2}-1$-simplex with $m>m_{\mathfrak{P}}=\frac{(d+2)(d-1)}{2}(d$ is the dimension of the Hilbert space we are considering). As a consequence for pure states $\sum_{i=1}^{d^{2}} p_{i}^{2}=\frac{2}{d(d+1)}$, where $p_{i}$ is the probability of obtaining result $i$ when measuring the SIC-POVM $\left\{E_{i}\right\}_{i=1}^{d^{2}}$.

The final objective of this work is to understand in physical, not purely mathematical, terms why $\mathcal{Q}$ has that structure. Why are the pure states situated on a sphere? Why is this sphere tangent to some of the facets of our simplex $\Delta_{d^{2}}$ ? Why are these facets precisely those of dimension $\frac{(d+2)(d-1)}{2}$ ?. 
I think that trying to answer this questions we will have a deeper understanding of Quantum Foundations, and therefore of our world.

Acknowledgment. I want to acknowledge Dr. Chris Fuchs, that kindly give me his endorsement to submit papers to the arXiv.

\section{REFERENCES}

[1] D. M. Appleby, A. Ericsson, And C. A. Fuchs, Properties of qbist state spaces. http://arxiv.org/abs/0910.2750.

[2] I. Bengtsson and K. Życzkowski, Geometry of Quantum States, Cambridge University Press, 2006.

[3] C. Fuchs And R. Schack, A quantum-bayesian route to quantum-state space, Foundations of Physics, 41 (2011), pp. 345-356. 10.1007/s10701-009-9404-8.

[4] C. A. Fuchs, Quantum mechanics as quantum information (and only a little more). http://arxiv.org/abs/quant-ph/0205039, 2002.

[5] M. KeYL, Fundamentals of quantum information theory, Physics Reports, 369 (2002), pp. 431 $-548$.

[6] G. Kimura, The bloch vector for n-level systems, Physics Letters A, 314 (2003), pp. 339 349.

[7] G. Kimura1 and A. Kossakowski, The bloch-vector space for n-level systems: the sphericalcoordinate point of view, Open Systems \& Information Dynamics, 12 (2005), pp. 207-229.

[8] J. M. Renes, R. Blume-Kohout, A. J. Scott, and C. M. Caves, Symmetric informationally complete quantum measurements, Journal of Mathematical Physics, 45 (2004), pp. 2171-2180.

E-mail address: joseirs@hotmail.com 